Supporting Information for the manuscript

Hierarchical Colloidosomes with a Highly Ordered and Oriented

Arrangement of Gold Nanorods via Confined Assembly at the

\title{
Emulsion Interface
}

Xuan Yue, ${ }^{1,2}$ Xuejie Liu, ${ }^{1,2}$ Nan Yan, ${ }^{* 1}$ and Wei Jiang*1,2

${ }^{1}$ State Key Laboratory of Polymer Physics and Chemistry, Changchun Institute of

Applied Chemistry, Chinese Academy of Sciences, Changchun 130022, China

${ }^{2}$ University of Science and Technology of China, Hefei 230026, China

*Corresponding authors

*E-mail: nyan@ciac.ac.cn;

*E-mail: wjiang@ciac.ac.cn 


\section{SUPPORTING FIGURES:}
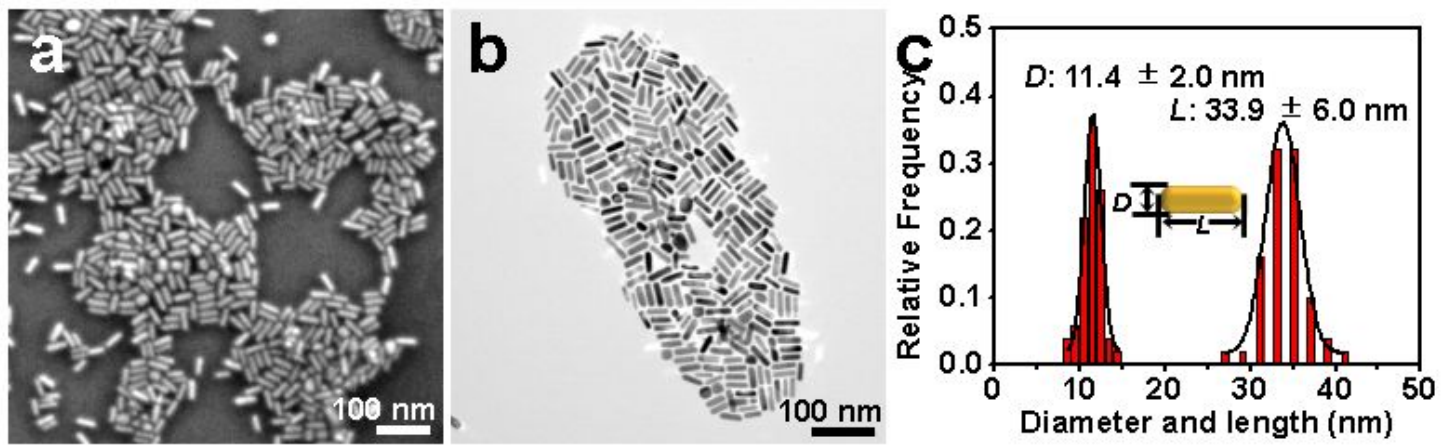

Figure S1. SEM (a) and TEM (b) images of the synthesized $\mathrm{PS}_{2 \mathrm{k}}$-tethered AuNRs-1, respectively. (c) The diameter and length distribution histograms of AuNRs-1 are obtained from the statistics of 100 AuNRs by TEM analysis software.
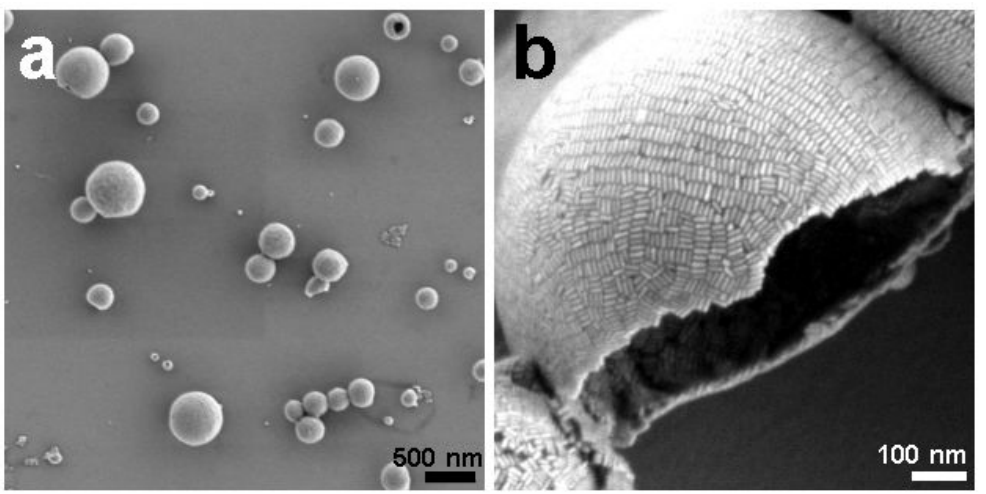

Figure S2. (a) SEM image of hierarchical AuNR colloidosomes at low magnification.

(b) SEM image of a broken AuNR colloidosome. 

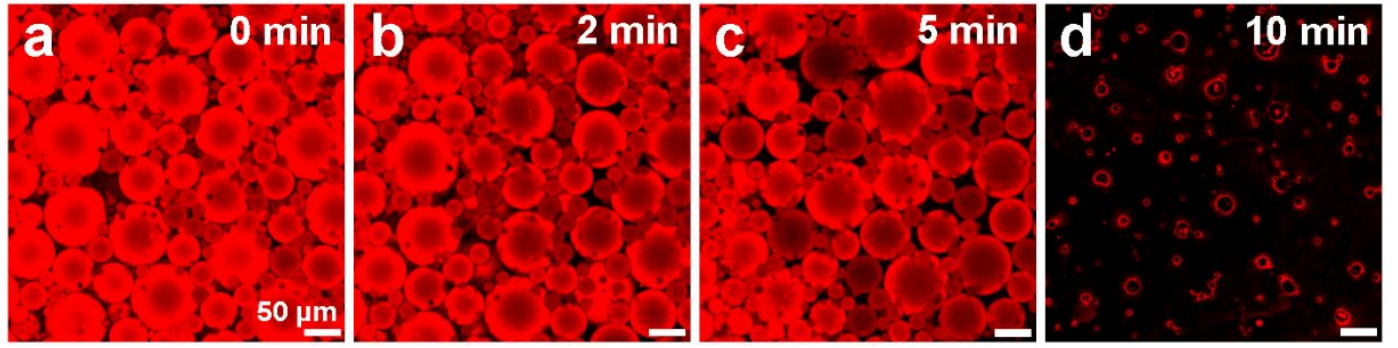

Figure S3. CLSM images showing the size evolution of the emulsion droplets with the evaporation of chloroform. Initial time (i.e., $0 \mathrm{~min}$ ) is regarded as the moment of capturing the droplet by CLSM after rapidly adding the freshly prepared emulsion onto a glass sheet. With the evaporation of emulsion droplets (i.e., $2 \mathrm{~min}, 5 \mathrm{~min}$ and 10min), HD phase coalesces forming liquid core and the AuNRs@PS $\mathrm{PS}_{2 \mathrm{k}}$ building blocks and Nile Red molecules can spontaneously migrate to the emulsion interface, and eventually redring assemblies are formed. The scale bar in the first image applies to the others. 


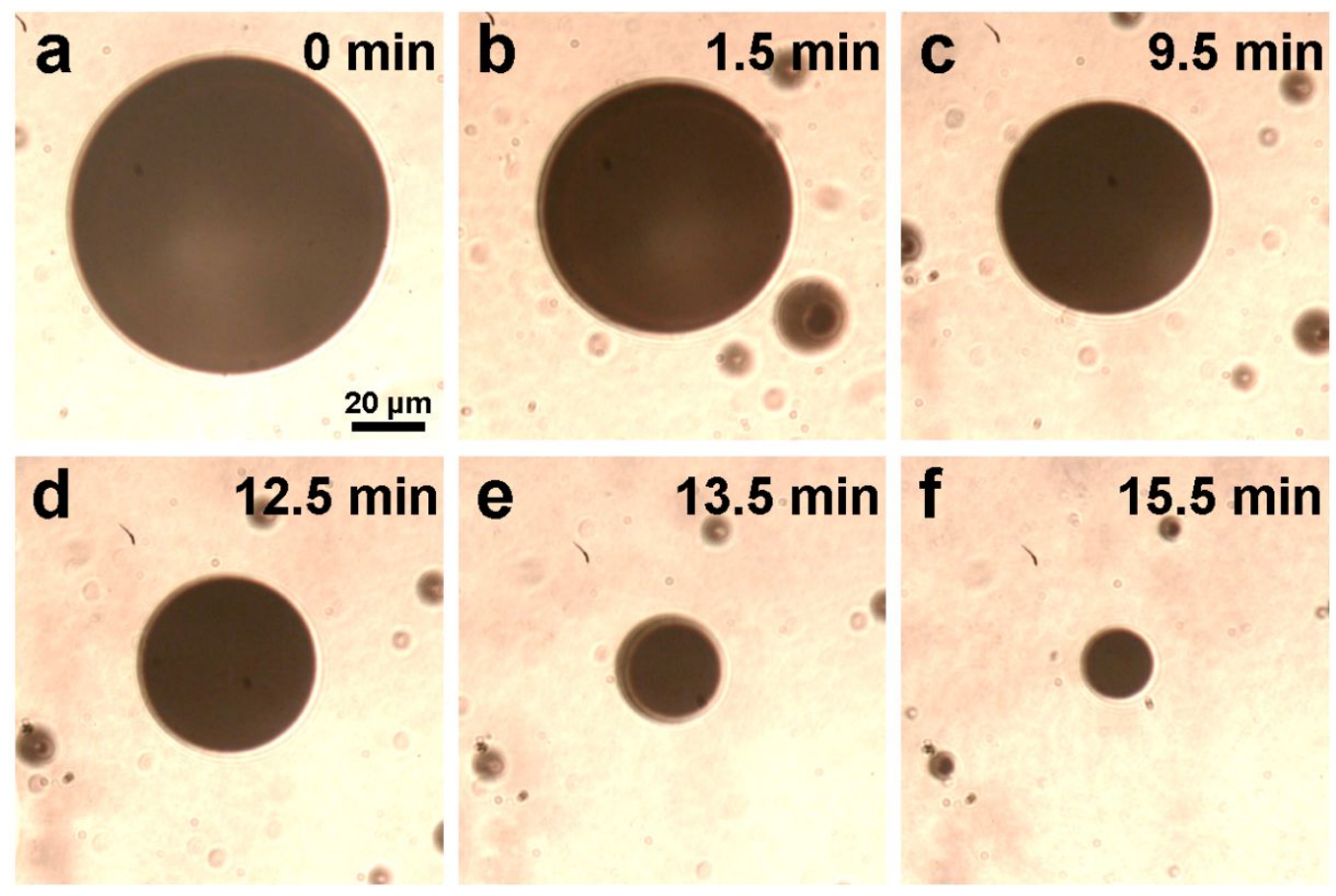

Figure S4. Optical microscopy (OM) images showing the size evolution of the emulsion droplets with the evaporation of chloroform. Initial time (i.e., $0 \mathrm{~min}$ ) is regarded as the moment of capturing the droplet by OM after rapidly adding the freshly prepared emulsion onto a glass sheet. With the evaporation of emulsion droplets, the size of the droplets decreases dramatically. The scale bar in the first image applies to the others.
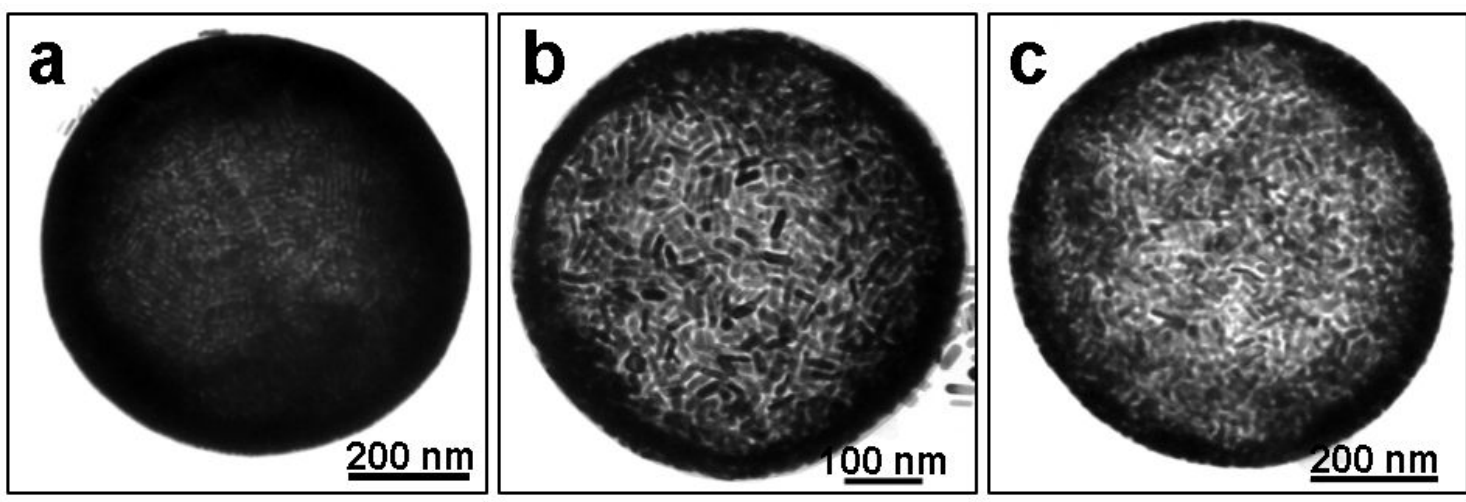

Figure S5. TEM images of AuNR colloidosomes fabricated from AuNRs@PS building blocks with different molecular weight of PS ligands. (a) $\mathrm{PS}_{2 \mathrm{k}}$, (b) $\mathrm{PS}_{5 \mathrm{k}}$, (c) $\mathrm{PS}_{20 \mathrm{k}}$. 

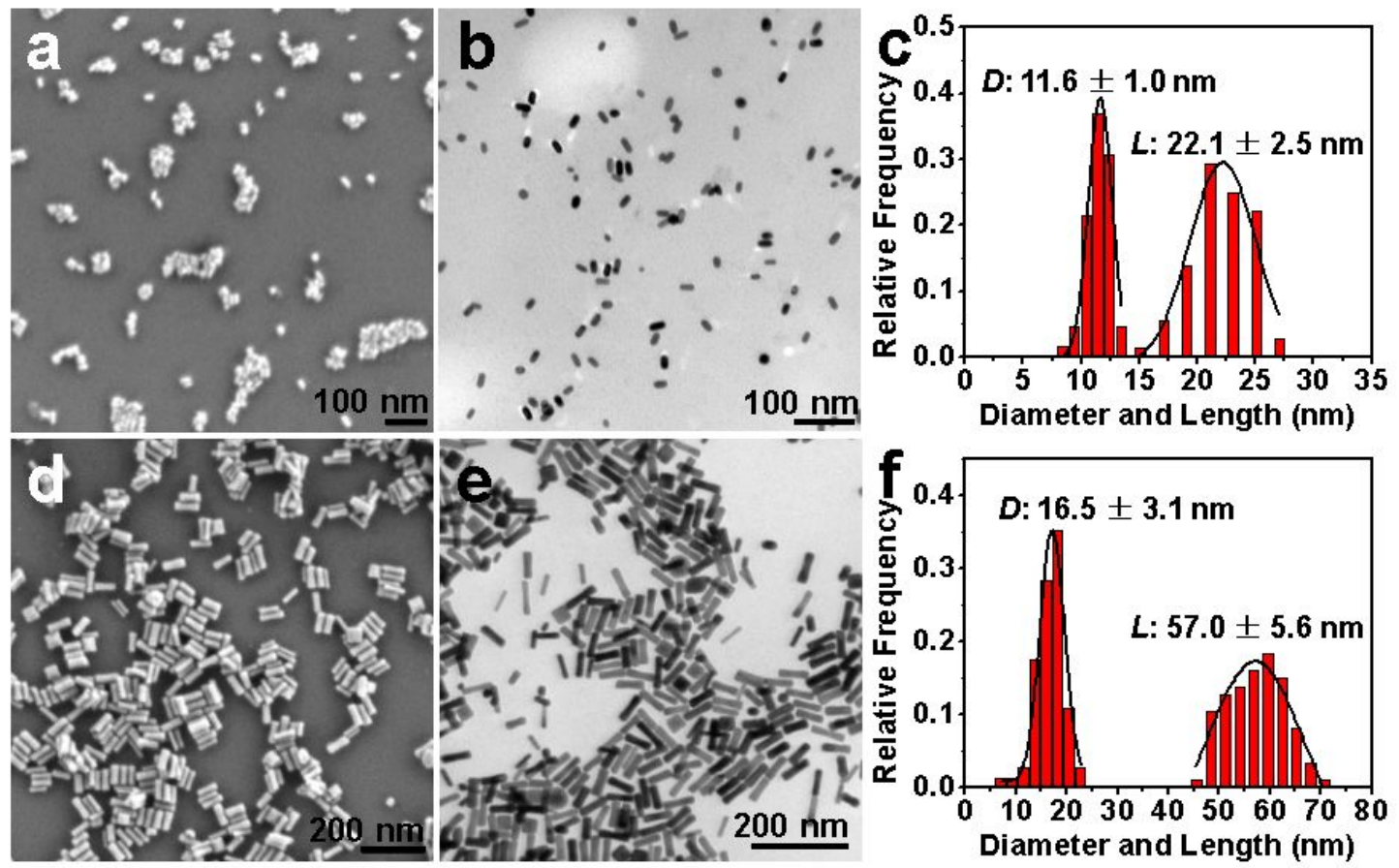

Figure S6. SEM (a, d) and TEM (b, e) images of the synthesized AuNRs-2 and AuNRs-

3, respectively. (c, f) The diameter and length distribution histograms of AuNRs-2 and AuNRs-3 are obtained from the statistics of 100 AuNRs by TEM analysis software, respectively.

Table S1. The synthesized AuNRs with different lengths $(L)$.

\begin{tabular}{cccc}
\hline AuNRs & AuNRs-2 & AuNRs-1 & AuNRs-3 \\
\hline$L(\mathrm{~nm})$ & $22.1 \pm 2.5 \mathrm{~nm}$ & $33.9 \pm 6.0 \mathrm{~nm}$ & $57.0 \pm 5.6 \mathrm{~nm}$ \\
\hline
\end{tabular}

Table S2. The mean square end-to-end distances $\left(R_{0}\right)$ of the tethered PS ligands on the AuNRs with different molecular weight.

\begin{tabular}{cccc}
\hline PS ligands & $\mathrm{PS}_{2 \mathrm{k}}$ & $\mathrm{PS}_{5 \mathrm{k}}$ & $\mathrm{PS}_{20 \mathrm{k}}$ \\
\hline$R_{0}(\mathrm{~nm})$ & 3.0 & 4.5 & 9.5 \\
\hline
\end{tabular}


Table S3. The $L / R_{0}$ value calculated from the AuNRs with different length and PS ligands with different molecular weight.

\begin{tabular}{cccc}
\hline$L$ & $L / R_{0}\left(\mathrm{PS}_{2 \mathrm{k}}\right)$ & $L / R_{0}\left(\mathrm{PS}_{5 \mathrm{k}}\right)$ & $L / R_{0}\left(\mathrm{PS}_{20 \mathrm{k}}\right)$ \\
\hline$L_{2}$ & 7.4 & 4.9 & 2.3 \\
$L_{1}$ & 11.3 & 7.5 & 3.6 \\
$L_{3}$ & 19.0 & 12.7 & 6.0 \\
\hline
\end{tabular}

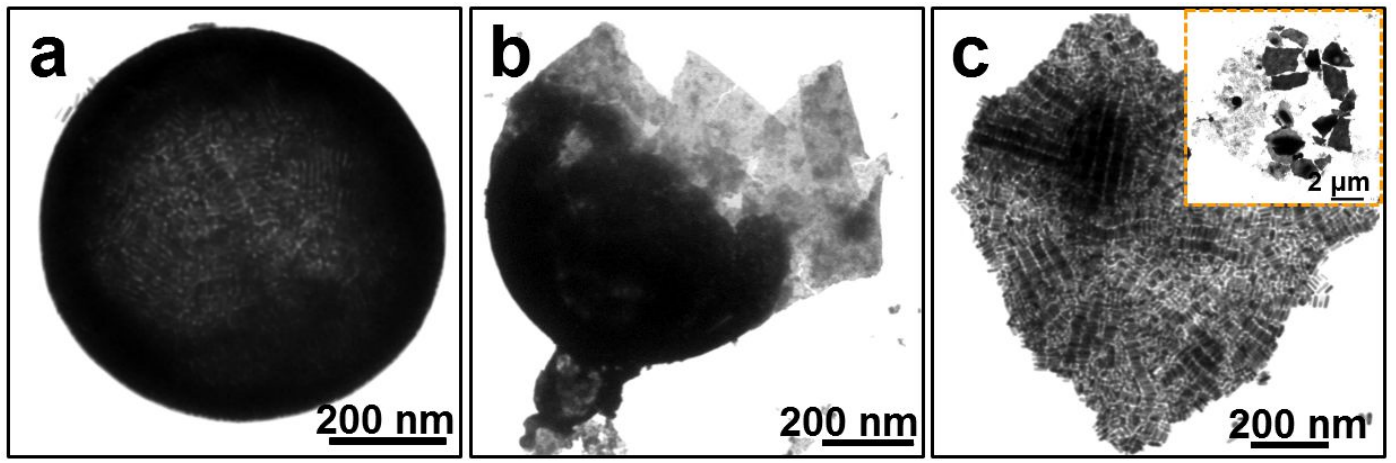

Figure S7. TEM images of AuNRs@PS $\mathrm{PS}_{2 \mathrm{k}}$ assembled superstructures prepared by varying the volume ratios of AuNRs@PS $\mathrm{PS}_{2 \mathrm{k}}$ solution to HD solution. (a) 7:3, (b) 5:5, and (c) 3:7. The inset in (c) is the TEM image of 2D sheet-like superlattices at low magnification.

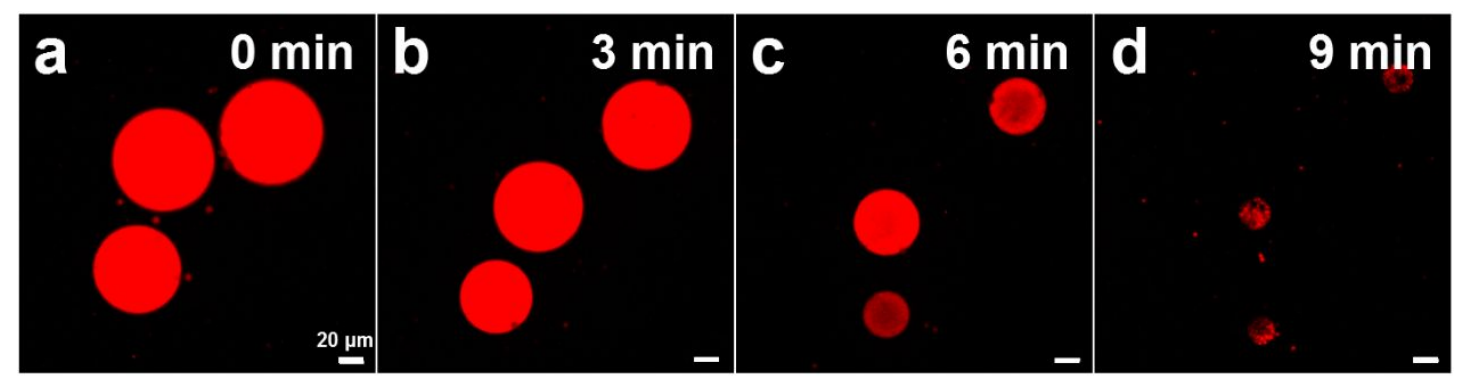

Figure S8. CLSM images showing the formation process of $2 \mathrm{D}$ sheet-like AuNRs@PS $\mathrm{PS}_{2 \mathrm{k}}$ superlattices with the evaporation of chloroform. 
Table S4. Estimation of the graft density of PS ligands grafted on the AuNRs.

\begin{tabular}{cccc}
\hline PS ligands & $W_{P S}$ vol\% & $N_{\text {grafts per nanorod }}$ & Graft density $\left(\right.$ chains $\left./ \mathrm{nm}^{2}\right)$ \\
\hline PS $_{2 \mathrm{k}}$ & 8.2 & 1790 & 1.26 \\
PS $_{5 \mathrm{k}}$ & 12.4 & 1135 & 0.80 \\
PS $_{20 \mathrm{k}}$ & 25.8 & 697 & 0.49 \\
\hline
\end{tabular}

Note: $W_{P S}$ is the weight fraction of the polymer ligands. $N_{\text {grafts per nanorod }}$ is the average number of polymer ligands on per nanorod.

The size of an $\mathrm{Au}$ atom is $0.017 \mathrm{~nm}^{3}$. The number of $\mathrm{Au}$ atom $\left(N_{A u}\right.$ atom $)$ in AuNR $(\sim 11.4 \times 33.9 \mathrm{~nm})$ can be calculated using Equation $\mathrm{S} 1$, where $r$ is the radius and $L$ is the length of the AuNRs. The result is 203437 gold atoms per nanorod and therefore the molar mass $\left(M_{A u N R}\right)$ of the AuNR is $197 N_{A u \text { atom. }}$ The average number of polymer ligands can be calculated by Equation S2, where, $W_{A u N R}$ is the weight fraction of AuNR and $M_{P S}$ is the molar mass of PS ligands. The graft density is calculated using Equation S3.

$N_{\text {Au atom }}=\frac{V_{\text {AuNR }}}{V_{\text {Au atom }}}=\left(\frac{\pi r^{2} L}{V_{\text {Au atom }}}\right)$ Equation S1

$N_{\text {grafts per nanorod }}=\left(\frac{W_{P S} / M_{P S}}{W_{A u N R} / M_{A u N R}}\right)$

Equation S2

Graft density $=N_{\text {grafts per nanorod }}\left(2 \pi r^{2}+2 \pi r L\right)$ Equation S3 